\title{
Pulmonary nodules incidentally detected on attenuation-correction CT scans in MPI
}

We agree with the conclusions of $\mathrm{He}$ et al. ${ }^{1}$ regarding the need for a greater degree of training for specialists primarily aligned with adult internal medicine, rather than radiology, to ensure appropriate reporting of important incidental findings on attenuation-correction CT images in myocardial perfusion imaging (MPI). In Australia, MPI studies are reported by Nuclear Medicine specialists who are accredited by the Australasian Association of Nuclear Medicine Specialists (AANMS). To gain this accreditation, it is mandatory to either have formal radiology training through the Royal Australasian and New Zealand College of Radiologists or, if trained primarily through the Royal Australasian College of Physicians (in adult internal medicine), to have undertaken a cross-sectional imaging course managed by AANMS. This ensures adequate maintenance of reporting standards. Perhaps this model could be extended to a wider geography?

Of particular concern is the poor inter-reader agreement between Cardiologists and Radiologists for pulmonary nodules in this study, in comparison to other incidental findings. Assessment for nodules is especially important given the potential to detect previously undiagnosed lung cancer in a small but significant percentage as described in our case series. ${ }^{2}$ It is interesting to note Cardiologists more frequently reported the presence of pulmonary nodules than their Radiologist counterparts, encompassing both nodules less than $10 \mathrm{~mm}$ (in diameter) and those greater. The degree of disagreement is disconcerting-especially for the larger nodules. Intuitively, we would have expected Radiologists to have a higher detection rate for suspicious pulmonary nodules. On the one hand, it is heartening

J Nucl Cardiol 2020;27:1055.

$1071-3581 / \$ 34.00$

Copyright (c) 2019 American Society of Nuclear Cardiology. from the viewpoint that there is such rigorous assessment for pulmonary nodules amongst non-Radiologists. On the other hand, does that imply cardiologists report some spurious nodules as radiologists may better be able to distinguish nodules from benign mimics such as atelectasis and intrapulmonary nodes?

\author{
Joseph C. Lee, MBBS, FRACP, FAANMS (D), ${ }^{a, b}$ \\ Francis T. Delaney, $M B B S^{c}$ \\ ${ }^{a}$ Department of Medical Imaging, The Prince \\ Charles Hospital, Chermside, QLD 4032, Australia \\ joseph.lee@health.qld.gov.au
}

${ }^{b}$ Faculty of Medicine, University of Queensland, Brisbane, Australia ${ }^{c}$ Department of Radiology, Mater Misericordiae University Hospital, Eccles St, Dublin D07 R2WY, Ireland

Disclosure Joseph Lee and Francis Delaney have no conflicts to declare.

\section{References}

1. He BJ, Malm BJ, Carino M, Sadeghi MM. Prevalence and variability in reporting of clinically actionable incidental findings on attenuation-correction CT scans in a veteran population. J Nucl Cardiol 2018. https://doi.org/10.1007/s12350-018-1232-9.

2. Delaney FT, Fong KM, Lee JC. Primary thoracic cancers incidentally detected on CT attenuation correction images during myocardial perfusion scintigraphy. Clin Lung Cancer 2018;19:e575-9.

doi:10.1007/s12350-019-01658-3

Publisher's Note Springer Nature remains neutral with regard to jurisdictional claims in published maps and institutional affiliations. 